\title{
Impacts of urban surface characteristics on spatiotemporal pattern of land surface temperature in Kunming of China
}

\author{
$\mathrm{XuChen}^{\mathrm{a}, \mathrm{b}, *}$, YapingZhang ${ }^{\mathrm{a}}$ \\ ${ }^{a}$ Faculty of Computer Science \& Information Technology, Yunnan Normal University, Kunming 650032, China; \\ ${ }^{\mathrm{b}}$ Computer\& Information Science Department, Southwest Forestry University, Kunming 650224, China
}

\begin{abstract}
To explore impacts of urban surface characteristics on spatiotemporal pattern of land surface temperature (LST), LST was retrieved from the thermal infrared band of satellite images and five indices were selected and extracted from remote sensing images in different time periods respectively acquired from 1992 to 2014. The correlation analysis (pixel by pixel) and linear regression analysis showed that although Normalized Difference Built-up Index (NDBI) and LST had the highest correlation coefficient, a combination of Modified Normalized Difference Water Index (MNDWI) and Normalized Difference Vegetation Index (NDVI) yielded the best regression results (the mean of Rsquared value increased 0.1 ). These results showed that both NDBI and NDVI-MNDWI would be acceptable indicators of LST, but NDVI-MNDWI could be better. Moreover, urban heat island (UHI) intensity (represented by LST) analysis showed that the highest UHI intensity appeared in the April, while the lowest UHI intensity index emerged in June. These results suggested that both UHI intensity and UHI intensity index might be closely related to land surface moisture. Furthermore, the regions of UHI intensity index greater than 0.5 were unchanged essentially from 2000 to 2014. So, these regions would be considered as key areas where the UHI could be focused on elimination.
\end{abstract}

Keywords : Urban heat island; Urban surface characteristics; Land surface temperature; Remote sensing

\section{Introduction}

Over the past decades, population growth and economic expansion have been the primary drivers of land use/cover change (LUCC) in the developing countries (Li et al., 2009). In China, the urbanization process has been accelerated from the late 1970s because of the launch of economic reforms (Luo \& Wei, 2009). The natural vegetation and agricultural land have been replaced by built-up areas (Van \& Bao 2010). A series of environmental problems have been induced in urban (Roy et al., 2009). Because increasing temperatures in the urban area may lead to significant ecological and social consequences, Urban heat island (UHI) effect has been a concern for much more than 40 years, and become a research hot topic in urban climatology and urban ecology (Chen et al., 2006; Li, Zhou, \& Ouyang, 2013). Generally, there are three types of urban heat islands: Surface heat islands (by measuring the infrared radiation emitted and reflected by surfaces, it is possible to identify the locations in a city where the surfaces are hottest); canopy layer heat islands (the canopy layer is the layer of air between the ground and treetops, or roofs of buildings, where most human activity takes place); and boundary layer heat islands (the boundary layer is located above the canopy layer). Canopy and boundary layer heat islands refer to air temperature (Oke, 1982; Li, Zhou, \& Ouyang, 2013). As a major parameter associated with surface radiation and energy exchange, Land surface temperature (LST) is able to modulate the air temperature of the layer immediately above the earth surface (Zhou \& Wang, 2011; Li, Zhou, \& Ouyang, 2013). Consequently, LST retrieved from infrared remote sensing imagery has been widely applied to study the spatial pattern of surface temperature UHI and its relationships with urban surface characteristics. Commonly, LUCC can affect the local thermal environment because each land use/cover type has its unique thermal, moisture, and optical spectral properties (Oke, 1982). However, little information of thermal characteristics on intra-class differences can be discovered by traditional urban land use/cover classification schemes (Xiao et al., 2007), because LST is subjected to several other factors, such as photosynthetic activity of vegetation, albedo, land cover fractions, soil moisture conditions and emissivity (Deng \& Wu, 2013; Li et al., 2011; Ghosh \& Joshi, 2014).

Therefore, remote sensing indices like Normalized Difference Vegetation Index (NDVI, Formula 11) (Rouse et al., 1973), Normalized Difference Built-up Index (NDBI, Formula 12) (Zha, Gao, \& Ni, 2003), Normalized Difference Bareness Index (NDBaI, Formula 13) (Chen et al., 2006), Normalized Difference Water Index (NDWI, Formula 14) (Gao, 1996) and Modified Normalized Difference Water Index (MNDWI, Formula 15) (Xu, 2006) have been used to research on relationships between LST and urban surface characteristics considerably. The NDVI is widely used to describe the general vegetation conditions and greenness of an area (Chen \& Brutsaert, 1998; Weng, Lu, \& Schubring, 2004). A higher NDVI indicates the higher probability of vegetation (Han, Chen, \& Feng, 2015). The NDBI can reveal the built-up and barren land of urban areas (Chen et al., 2006; Zhou \& Wang, 2011). High NDBI 
values generally signify areas with intensive urban development (Zhou \& Wang, 2011). Based on reflected near-infrared radiation and visible green light, NDWI not only can enhance the open water features but also is sensitive to changes in liquid water content of vegetation canopies ( $\mathrm{Zha}$, Gao, \& Ni, 2003; Gao, 1996). Because water areas often reveal remarkable differences in thermal characteristics, MNDWI is sensitive to water bodies too. And the built-up land noise on water can be removed by comparing NDWI with MNDWI in the urban areas (Xu, 2008a; Zhou \& Wang, 2011). Furthermore, there are lots of others spectral indices, such as normalized difference impervious surface index (NDISI, Xu, 2008b), index-based built-up index (IBI, Xu, 2008a), and the fractional vegetation cover or proportion of vegetation (Fv or Pv, Carlson \& Ripley, 1997; Wu et al., 2014; Vlassova et al., 2014). Especially, impervious surfaces (IS) are primarily associated with transportation (streets, highways, parking lots and sidewalks) and building rooftops in remote sensing (Yuan \& Bauer, 2007; Zhang, Odeh, \& Han, 2009). As one of the most important land-cover types, impervious surface area (ISA) has been used to quantify the degree of urbanization (Yuan \& Bauer, 2007). So ISA can affect urban surface temperatures by altering the sensible and latent heat fluxes (Yang et al., 2003; Xiao et al., 2007). Otherwise, the percent of ISA can indicate the development level of urban space (Zhang, Odeh, \& Han, 2009).

Although the relationships between LST and urban surface characteristics can be quantitatively studied by methods described above, the impact of background noises (such as, bare soils, plants or water bodies) and spatiotemporal changes cannot be avoided (Ma, Kuang, \& Huang, 2010). For example, NDVI may influence the results of land surface UHI analysis because it is susceptible to seasonal variations (Zhang, Odeh, \& Han, 2009). Moreover, as well known, the relationship between NDVI and LST is nonlinear. The surface radiant temperature of predominantly bare ground surfaces exhibits larger variation than the densely vegetated LUCC types (Chen et al., 2006). Therefore it is not sufficient to quantitatively study UHI by only using NDVI (Zhang, Odeh, \& Han, 2009). Similarly, because of the complexity of urban landscapes and the limitation of remotely sensed data, the identification of ISA is very difficult (Zhang, Odeh, \& Han, 2009). At the same time, interpreting thermal data and images of temperature distribution over an area is difficult for many complex factors involved (Weng, Lu, \& Schubring, 2004). The most influential factors for controlling the UHI are the distribution of surface cover characteristics, and urban morphology, such as building materials, geometry, and density (Li, Zhou, \& Ouyang, 2013). Each component surface in urban landscapes exhibits a unique radiative, thermal, moisture, and aerodynamic properties, and relates to their surrounding site environment (Weng, Lu, \& Schubring, 2004). Higher levels of latent heat fluxes are more representative of areas characterized by significant vegetation cover (e.g., forest and grass-land areas) in comparison to areas with sparse or no vegetation cover and low surface moisture availability (such as built-up and bare-soil types) (Zhang, Odeh, \& Han, 2009). Moreover, the intensity of UHI is influenced by the change of urban surface characteristics, such as spatial extent and composition of vegetation or built-up areas and the spatial arrangement of the urban land uses (Martilli 2014; Debbage \& Shepherd 2015). So, the relationship between LST and urban surface characteristics needs further calibration and investigation (Weng, Lu, \& Schubring, 2004). Otherwise, multi-temporal studies of UHI over four seasons need to be focused by using different satellite data (Zhang, Odeh, \& Han, 2009), and some technologies used to analyze the urban surface characteristics and urban thermal environment also need to be further improved (Ma, Kuang, \& Huang, 2010).

Specifically, for exploring the impact of urban surface characteristics on spatiotemporal pattern of land surface temperature in a rapidly urbanization city, Kunming of China during the period 19922014, LST and Five indices (NDVI, NDBI, NDBaI, NDWI and MNDWI) were retrieved from remote sensing images in different time periods respectively. The objectives of this study were: (1) to quantitatively compare patterns and intensities of LST; (2) to quantitatively investigate the relationships between the urban surface characteristics and LST; (3) to study how these relationships has changed over time and try to find out which one is relatively stable.

\section{Study area and dataset}

\subsection{Study area}

Kunming, located from $102^{\circ} 10^{\prime}$ to $103^{\circ} 40^{\prime} \mathrm{E}$ and $24^{\circ} 23^{\prime}$ to $26^{\circ} 33^{\prime} \mathrm{N}$, is in the north-central Yunnan province, China (Fig. 1). It is situated on the Yunnan-Guizhou Plateau with elevation ranging from $1500 \mathrm{~m}$ to $2800 \mathrm{~m}$, and surrounded by mountains. The city is characterized by a subtropical highland climate, which is warm and humid in summer, as well as cold and dry in winter. Mean annual precipitation and sun exposure are around $1000 \mathrm{~mm}$ and about 2250 hours respectively (Zhou \& Wang, 2011). Kunming has experienced rapid development in the last two decades, and become an international commercial hub for South and Southeast Asia. A great of green space has lost in the 
suburban areas accompanied by rapid urbanization and fast economic growth (Cai, 2007). In 2011, a new district (ChengGong) was established in Kunming and urbanized area further expanded. Therefore, we selected the metropolitan area of Kunming, an area of $2622 \mathrm{~km}^{2}$, consisting of urban area (dominated by impervious land), urban fringe (combination of impervious land and agriculture land), and suburban area (dominated by forest land) as the study area.

\subsection{Datasets}

The satellite images collected by Landsat instruments, including TM, ETM+, and OLI were downloaded from United States Geological Survey (USGS) Earth Resource Observation and Science (EROS) Center archive (http://earthexplorer.usgs.gov/) and the Geospatial Data Cloud of China (http://www.gscloud.cn/), which had resampled into 30m for all bands and corrected the radiometric, geometrical distortions and terrain of the images to a quality level of $1 \mathrm{~T}$ before delivery. Through visual examination, because of all data covered by cloud in July, cloud free images over 22 years were collected between January and December except July from 1992 to 2014. The temporal distribution of the data was listed in Table 1. Air temperature and precipitation data were obtained from the National Climate Center of China (http://ncc.cma.gov.cn/). Finally each of the satellite dataset was clipped to the extent of study area using a vector layer of administrative region.

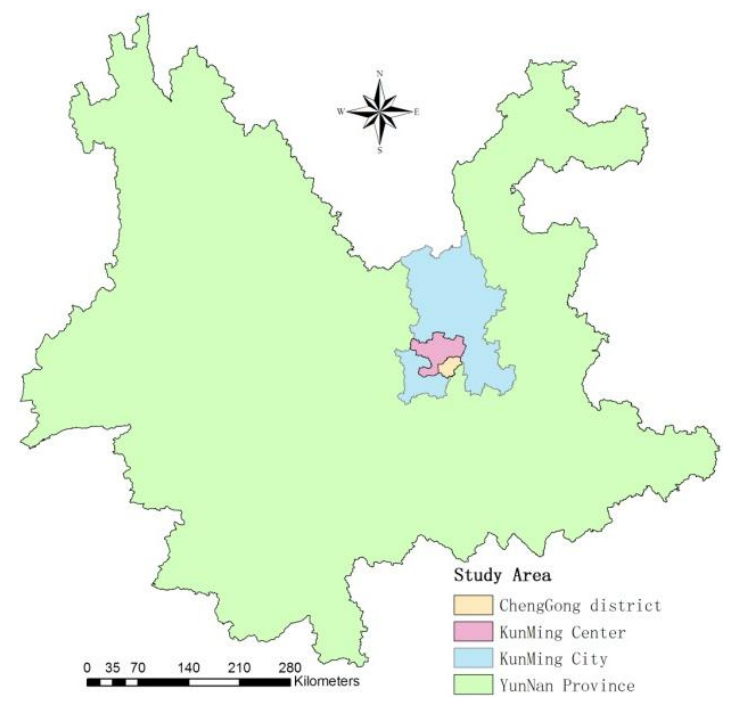

Fig.1. Study area.

Table 1 Temporal distribution of the data.

\begin{tabular}{ccccccccc}
\hline Date & Sensor & Time & Date & Sensor & Time & Date & Sensor & Time \\
\hline $16 / 8 / 1992$ & TM & $10: 57$ & $2 / 3 / 2001$ & TM & $11: 15$ & $1 / 4 / 2006$ & TM & TM \\
$1 / 9 / 1992$ & TM & $10: 57$ & $14 / 6 / 2001$ & ETM+ & $11: 24$ & $19 / 5 / 2006$ & $11: 27$ \\
$25 / 12 / 1993$ & TM & $10: 56$ & $30 / 4 / 2002$ & ETM+ & $11: 23$ & $24 / 3 / 2009$ & TM & TM \\
$16 / 1 / 1996$ & TM & $10: 38$ & $7 / 10 / 2002$ & ETM+ & $11: 23$ & $3 / 11 / 2009$ & $11: 25$ \\
$24 / 4 / 2000$ & ETM+ & $11: 24$ & $9 / 4 / 2003$ & TM & $11: 09$ & $20 / 4 / 2013$ & OLI/TIRS \\
$15 / 9 / 2000$ & ETM+ & $11: 24$ & $10 / 3 / 2004$ & TM & $11: 14$ & $2 / 2 / 2014$ & OLI/TIRS & $11: 36$ \\
$26 / 11 / 2000$ & TM & $11: 14$ & $25 / 2 / 2005$ & TM & $11: 21$ & $23 / 4 / 2014$ & OLI/TIRS & $11: 34$ \\
\hline
\end{tabular}

\section{Methodology}

ENVI5.1/IDL8.3 and Arcgis10.2 were used to process the all satellite images, and SPSS21 was used to carry out Statistical analyses. 


\subsection{Image preprocessing}

We used Radiometric Calibration tool in ENVI5.1 to calibrate image data to radiance, reflectance, and brightness temperatures, following the approach described in ENVI5.1 help document.

\subsubsection{Radiance}

The raw digital numbers (DNs) were converted to top-of-atmosphere (TOA) radiance if the image has gains and offsets for each band. ENVI reads these values from the Landsat metadata and radiance is computed using the following equation:

$$
L_{\lambda}=\text { gain } * D N+\text { offset }
$$

\subsubsection{Reflectance}

The raw digital numbers (DNs) were converted to TOA reflectance if the image has gains, offsets, solar irradiance, sun elevation, and acquisition time defined in the Landsat metadata. ENVI reads these values from metadata and reflectance is computed using the following equation:

$$
\rho_{\lambda}=\frac{\pi L_{\lambda} d^{2}}{E S U N_{\lambda} \sin \theta}
$$

Where:

$L_{\lambda}=$ Radiance

$d=$ Earth-sun distance

$E S U N_{\lambda}=$ Solar irradiance

$\theta=$ Sun elevation in degrees

With Landsat- 8 files, the reflectance gains and offsets have been scaled by the sine of the sun elevation.

\subsection{Land surface temperature retrieved}

\subsubsection{Brightness Temperature}

The raw digital numbers (DNs) were converted to TOA brightness temperature (i.e., blackbody temperature, TB) if the image has gains, offsets, and $\mathrm{K} 1$ and $\mathrm{K} 2$ calibration constants specified in the Landsat metadata. ENVI reads these values from metadata and brightness temperature (in Kelvin) is computed as follows:

$$
T=\frac{K 2}{\ln \left(\frac{K 1}{L_{\lambda}}+1\right)}
$$

This option is only available for Landsat-8, ETM+, and TM thermal imagery in ENVI. Especially, we select the band 10 to retrieval the LST for Landsat-8, because National Aeronautics and Space Administration (NASA) suggests not to use band 11 for surface temperature retrieval (Vlassova et al. 2014) and Yu, Guo, \& Wu (2014) found that band 10 has higher accuracy than band 11.

\subsubsection{Land surface temperature}

The temperature values obtained above are referenced to a black body. To retrieve the LST, the brightness temperature needs to be corrected according to the real object properties. Therefore, corrections for land surface emissivity (LSE or $\varepsilon$ ) become necessary according to the nature of land cover (Weng, Lu, \& Schubring, 2004; Li et al., 2009; Ma, Kuang, \& Huang, 2010; Zhou \& Wang, 2011; Ghosh \& Joshi, 2014). It is suggested that the emissivity values should be more detailed considering the nature of land cover types ( $\mathrm{Li}$ et al., 2009). The emissivity corrected surface temperature is computed as follows (Artis \& Carnahan, 1982):

$$
T_{S}=\frac{T_{b}}{1+\left(\frac{\lambda \times T_{b}}{\alpha}\right) l n \varepsilon}
$$

As the obtained surface radiant temperature is in Kelvin, the radiant temperature should be revised by adding the absolute zero (approximately $-273.15^{\circ} \mathrm{C}$ ):

$$
T_{S}=\frac{T_{b}}{1+\left(\frac{\lambda \times T_{b}}{\alpha}\right) \ln \varepsilon}-273.15
$$




\subsubsection{Land surface emissivity}

Land surface emissivity (LSE) is a key parameter in LST retrieval model (Yang et al., 2014). It is a measure of the ability of a surface to emit energy by radiation which has a great influence on LST retrieval (Yang et al., 2014). Because of the high level of correlation between NDVI and LSE, NDVI can be used to estimate the emissivity of the surface (Snyder et al., 1998; Zhang, Odeh, \& Han, 2009). Normally, the surface emissivity $\varepsilon$ is determined for three cases using the NDVI Thresholds Method (Sobrino, Raissouni, \& Li, 2001; Sobrino, Jime'nez-Muñz, \& Paolini, 2004; Xiao et al., 2007; Yu, Guo, \& Wu, 2014; Vlassova et al., 2014; Wu et al., 2014). But LSE is more complicated than the variables described above. As each Landsat imagery pixel region $(30 \mathrm{~m} * 30 \mathrm{~m})$ could contain two or more land cover types and NDVI could be subject to seasonal variations, we used separation method of mixed pixels to estimate the LSE. Based on the previous studies (Snyder et al., 1998; Sobrino, Raissouni, \& Li, 2001; Sobrino, Jime'nez-Muñoz, \& Paolini, 2004; Xiao et al., 2007;Yu, Guo, \& Wu, 2014;Vlassova et al., 2014; Wu et al., 2014; Yang et al., 2014), we used k-Means to classify the all images, resulting in four land cover types as water areas, low NDVI areas (LNDVI, including urban, road, industrial district, bare land, rural residential area and low vegetation cover areas), middle NDVI areas (MNDVI, including grassland, arable land, bush and sparse vegetation cover areas) and high NDVI areas (HNDVI, including vegetative areas). As water body and HNDVI pixels are easy to be distinguished and relatively unitary, their emissivities are directly assigned as 0.995 and 0.986 , respectively (Zheng et al., 2010; Yang et al., 2014). LNDVI pixels could be regarded as the mixture of urban or bare land and vegetation. Similarly, MNDVI pixels could be regarded as the mixture of vegetation and soil. According to Zheng et al. (2010) and Yang et al. (2014), the emissivities of LNDVI areas and MNDVI areas are computed as Eq. 6 and Eq. 7, respectively.

$$
\begin{aligned}
\varepsilon_{t} & =0.9608420+0.0860322 P_{V}-0.0671580 P_{V}{ }^{2} \\
\varepsilon_{n} & =0.9643744+0.0614704 P_{V}-0.0461286 P_{V}{ }^{2}
\end{aligned}
$$

Where, $\mathrm{Pv}$ is the vegetation fraction estimated from a NDVI and is computed as follows (Choudhury et al., 1994; Gutman \& Ignatov, 1998; Vlassova et al., 2014):

$$
P_{V}=\frac{N D V I-N D V I_{S}}{N D V I_{V}-N D V I_{S}}
$$

In Eq. 8, NDVIv is NDVI value of pure vegetation area and is assigned 0.7; NDVIs is bare soil or non-vegetation covering area and is assigned 0.05 . Therefore, when NDVI $>0.7$, it can be regarded as full vegetation cover, with both $\varepsilon_{\mathrm{t}}$ and $\varepsilon_{\mathrm{n}}$ being 0.986 ; when NDVI $<0.05$, it can be regarded as bare soil, with both $\varepsilon_{\mathrm{t}}$ and $\varepsilon_{\mathrm{n}}$ being 0.970 (Sobrino, Raissouni, \& Li, 2001; Sobrino, Jime'nez-Muñoz, \& Paolini, 2004); when $0.05 \leq \mathrm{NDVI} \leq 0.7$, emissivity can be calculated by Eq. 6 and 7. Based on the above method, the emissivity of each pixel can be calculated by combining NDVI and classification map (Yang et al. 2014).

\subsubsection{Classification validation}

Ideally, the reference data should be obtained through field surveys. However, the rapid land use changes make it difficult to conduct effective field observations. Fortunately, high-resolution images are accessible freely from Google Earth ${ }^{\mathrm{TM}}$ (http://earth.google.com/) and could be used as ground-truth for land cover classifications (Knorn et al., 2009). To assess the classification accuracy, high resolution historical images from Google Earth were used as the reference layers, and five hundred random sample points were generated in each image for comparing classification data and reference data (Feng et al., 2014). The overall accuracy was above $85 \%$ and the Kappa coefficient was above 0.78 for each image, which met the recommended value by Lucas, Frans, \& Wel (1994).

\subsection{Urban heat island intensity and Urban surface characteristics}

\subsubsection{UHI intensity (UHII) and UHI intensity index (UHIII)}

The urban heat island effect is a relative concept, and its degree indicates the temperature difference between the city and its natural/rural surroundings (Xu, Xie, \& Li, 2013). As the near-surface atmospheric water vapour content varies over time due to seasonality and inter-annual variability of the atmospheric conditions, it is inappropriate to directly compare temperature values represented by the LST between multiple periods (Zhang, Odeh, \& Han, 2009). Considering the difference in temperature and atmospheric conditions between acquisition times of the Landsat images, the UHI intensity (UHII) is used for comparing between two or more periods in this study, rather than looking at the absolute land surface temperature changes (Chen et al., 2006;Zhang, Odeh, \& Han, 2009). Followed the traditional approach, the UHI intensity can be calculated using a rural baseline (a heavily vegetated 
portion of the metropolitan area, HNDVI):

$$
U H I I=T_{o a^{-}} T_{m H}
$$

Where, $\mathrm{T}_{\mathrm{oa}}$ is temperature of other area and $\mathrm{T}_{\mathrm{mH}}$ is mean temperature of HNDVI.

The indicator of urban heat island intensity is calculated to reflect the spatial distribution of UHI intensity (Feng et al., 2014). This study introduced a relative intensity to indicate the UHII, which could also be called the urban heat island intensity index (UHIII):

$$
U H I I I=\frac{T_{o a}-T_{m H}}{T_{m H}}=\frac{U H I I}{T_{m H}}
$$

Larger value of the UHIII indicated greater effect of the corresponding land use change on the UHII.

\subsubsection{Urban surface characteristics}

Five remote sensing indices, NDVI, NDBI, NDBaI, NDWI and MNDWI were computed to characterize the urban surface of this study. These five indices are calculated as follows:

$$
\begin{gathered}
N D V I=\frac{R_{N I R}-R_{R E D}}{R_{N I R}+R_{R E D}} \\
N D B I=\frac{R_{M I R}-R_{N I R}}{R_{M I R}+R_{N I R}} \\
N D B a I=\frac{D_{M I R}-D_{T I R}}{D_{M I R}+D_{T I R}} \\
N D W I=\frac{R_{N I R}-R_{M I R}}{R_{N I R}+R_{M I R}} \\
M N D W I=\frac{R_{G R E E N}-R_{M I R}}{R_{G R E E N}+R_{M I R}}
\end{gathered}
$$

Where, RNIR is the reflectance in the near infrared band; RRED and RGREEN, respectively, stand for the reflectance in red and green bands; RMIR denotes the reflectance in the middle infrared; D represents digital number value $(\mathrm{DN})$ of corresponding bands, and DTIR represents DN of ETM+/band61, TM/band6 or TIRS/band10.

\subsection{Statistical analyses (Correlation and regression-Univariate regression and Multivariate regression)}

The relationship between LST and urban surface characteristics was examined using Pearson correlation analysis (Li, Zhou, \& Ouyang, 2013). Then, correlation analyses among the UHI intensity index, NDVI, NDBI, NDBaI, NDWI and MNDWI were used to establish a correlation model for further understanding this relationship (Xu, Xie, \& $\mathrm{Li}, 2013$ ). As the aim of regression is to learn the relationship between input and output variables by fitting a model to a set of training data (Ghosh \& Joshi, 2014), the resulting regression equation can be used to study the impact of urban surface characteristics on LST (Zhang, Odeh, \& Han, 2009). The linear regression analysis was employed again for each dataset in this study (Ma, Kuang, \& Huang, 2010). The strength of the correlation between LST and individual explanatory variables (i.e. each of the three remote sensing indices) was calculated firstly using univariate regression, and then multivariate regression was conducted to model the LST based on the combination of the explanatory variables (Zhou \& Wang, 2011). The multiple regression models were significant at 0.01level (Weng, Lu, \& Schubring, 2004).

\section{Results and discussion}

\subsection{Characteristics of LST, NDVI, NDBI, NDBaI, NDWI, MNDWI and UHIII}

Based on the above Formulas (1-15), images from 1992 to 2014 were used to extract the remote sensing information for the whole city of Kunming, including the LST, NDVI, NDBI, NDBaI, NDWI, MNDWI and UHIII (Fig. 2, Fig. 3). Higher LSTs distributed in the downtown, north, northeast, southeast and southwest areas of Kunming. Lower LSTs distributed in the west, east and northeast areas of Kunming. The heat islands (areas with relatively high temperatures) scattered in the area (bare land, semi-bare land and urban area were warmer than other areas). Except the intensity of the urban heat island slightly different, the spatial distribution of the UHI was consistent with land use/cover distribution in the same months, and UHI mainly concentrated in the north, northeast, east, and southeast and southwest (Fig. 2 (c), (f), (i)). Comparison with classification map (Fig. 2 (b), (e), (h)), UHI was mainly found in LNDVI and MNDVI areas. Water exhibited the lowest temperature with the 
minimum variation in all years because its relatively high thermal inertia reduced the heat difference, followed by the HNDVI region. Built-up and barren land showed the highest temperatures from 1992 to 2014 .

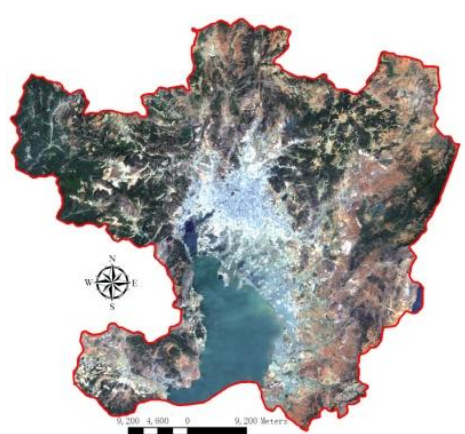

(a)

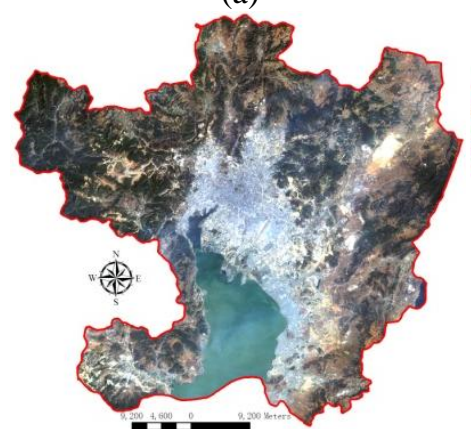

(d)

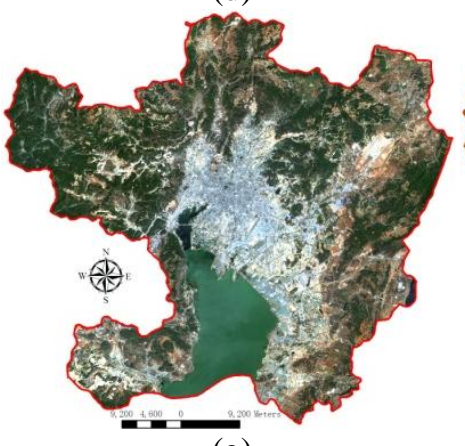

(g)

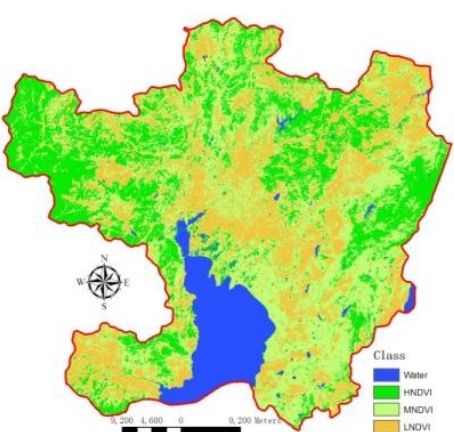

(b)

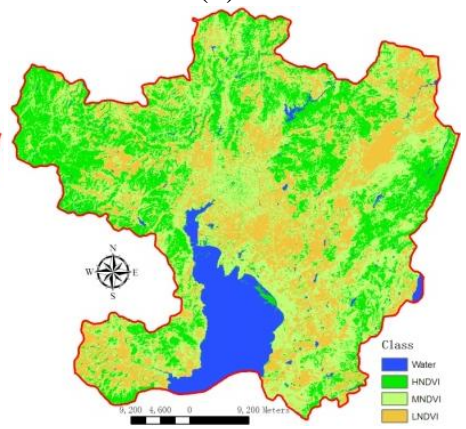

(e)

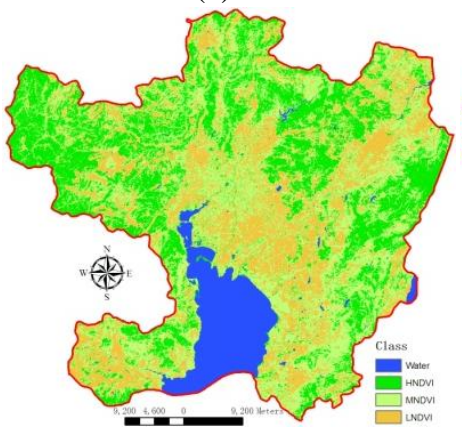

(h)

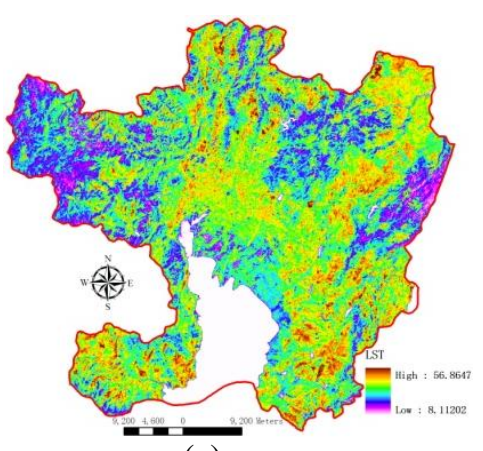

(c)

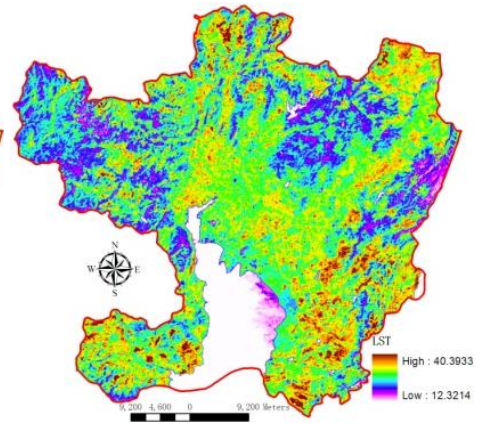

(f)

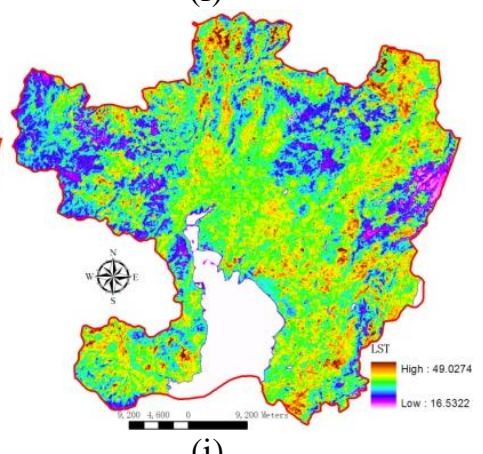

(i)

Fig.2. Spatial distributions of the LST in April, 2002 (Landsat7), March, 2009 (Landsat5) and April, 2013 (Landsat8).

The land surface or near land surface temperature can be affected by the characteristics (ranging from the bare ground to vegetation cover types of variable density) of land surface cover (Zhang, Odeh, \& Han, 2009). The spatial distributions of MNDWI, NDBaI, NDBI, NDVI, NDWI and UHIII in 2013 over the whole city of Kunming had a big difference, while each index was a good reflection of the main characteristics in study area (Fig. 3). Particularly, as NDBI and NDWI are the opposite indices each other, the distribution of the two methods is the same (Fig. 3 (c), (e)). At the same time, the distribution of UHIII and LST could also be found that was almost entirely consistent (Fig. 2 (i), Fig. 3 (f)). 


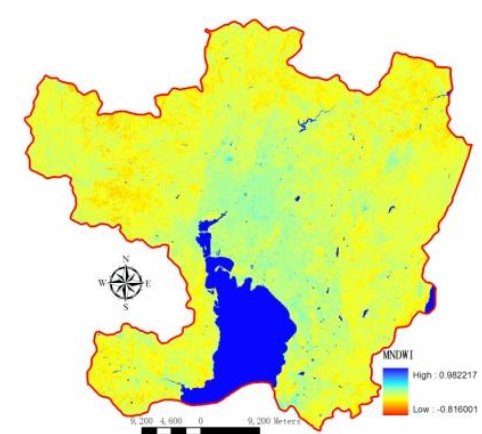

(a)

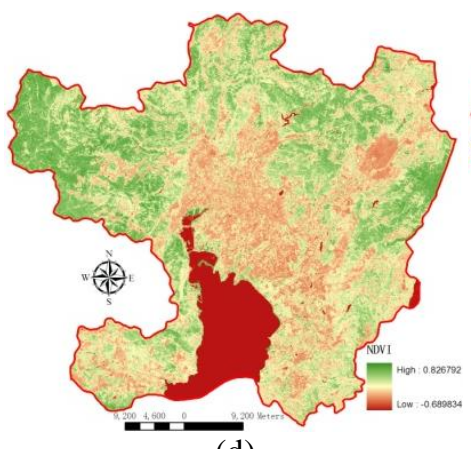

(d)

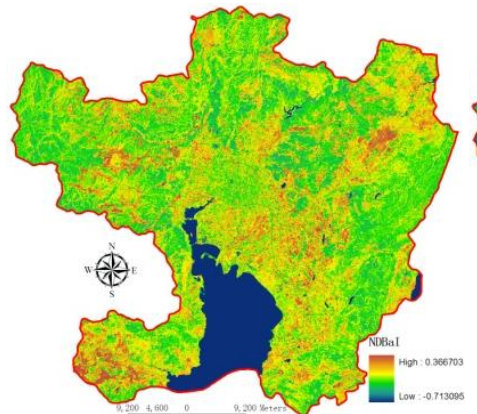

(b)

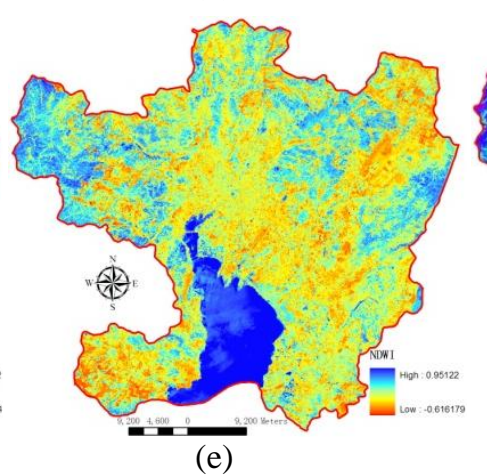

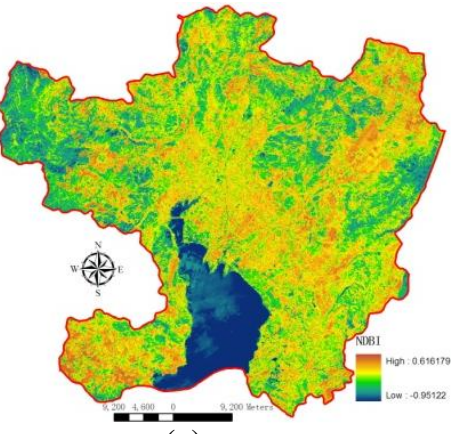

(c)

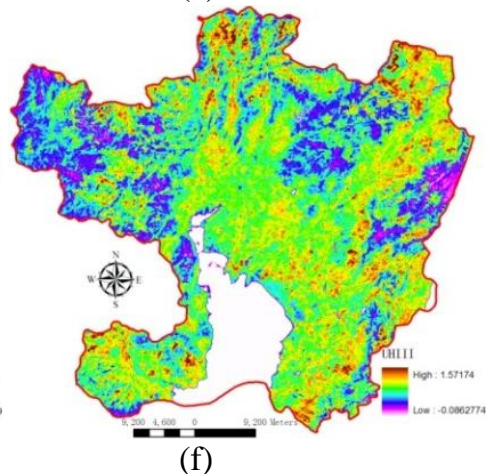

Fig.3. MNDWI, NDBaI, NDBI, NDVI, NDWI and UHIII in April, 2013 (Landsat8).

\subsection{Relationships between retrieval data and meteorological data}

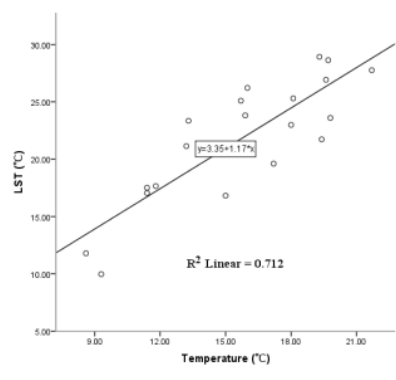

(a)

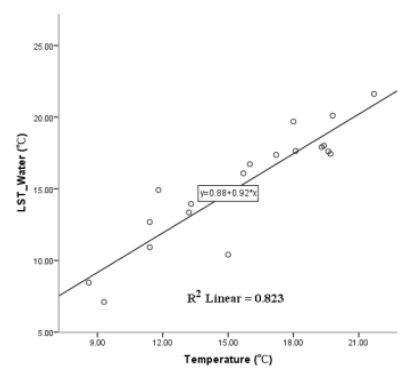

(b)

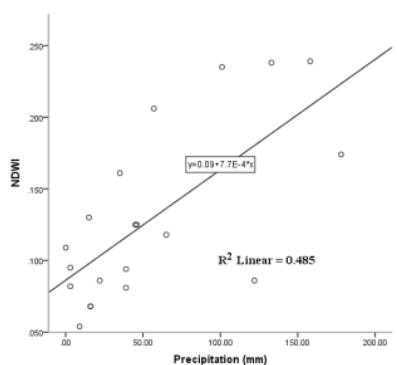

(c)

Fig.4. Regression coefficient between retrieval data and meteorological data.

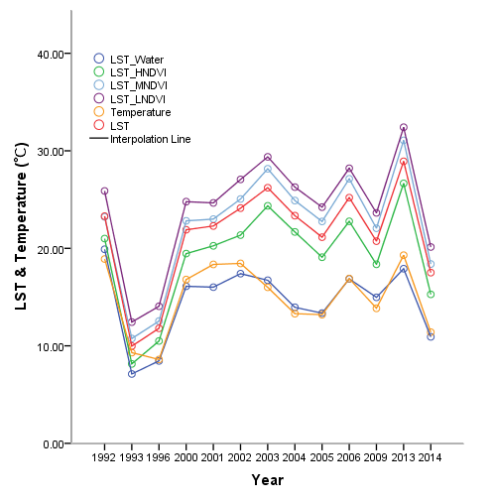

(a)

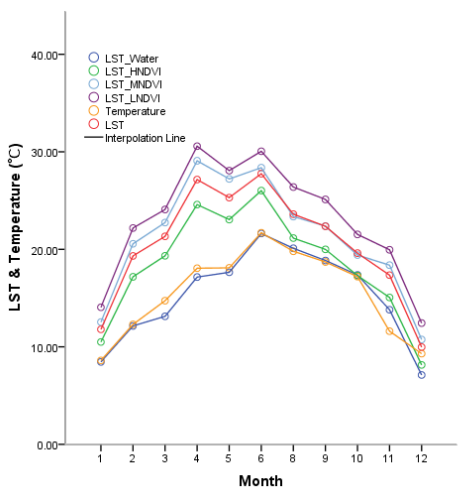

(b)

Fig.5. The changes of LST and temperature.

To validate the results of LST retrieval, correlation strength between LST and temperature was 
examined. There was a remarkable positive correlation between LST and Temperature, and the correlation coefficient was 0.844 (Fig. 4 (a), $P<0.01$ ). Particularly, the correlation coefficient increased from 0.844 to 0.907 (Fig. 4 (b), $P<0.01$ ) if the LST was replaced by surface temperature of water (STW) areas. Data indicated that the LST could represent changes of temperature, especially STW (Fig. 4, Fig. 5). Therefore, using the STW as reference of temperature was feasible. In addition, NDWI positively correlated with the precipitation, and the correlation coefficient was 0.696 (Fig. 4 (c), $P<0.01)$. This suggested that NDWI could depict the changes of rainfall to some extent and be used to substitute for the surface moisture availability.

\subsection{Comparison of our study and a similar study}

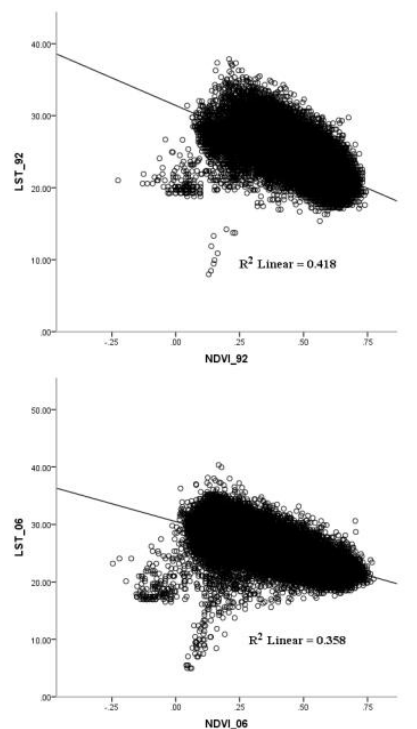

(a)
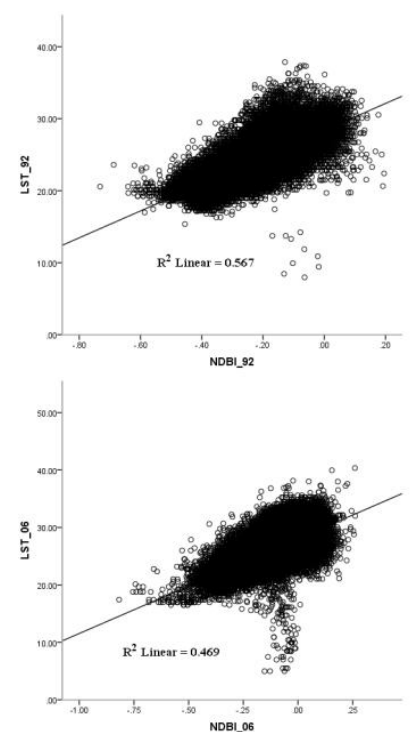

(b)

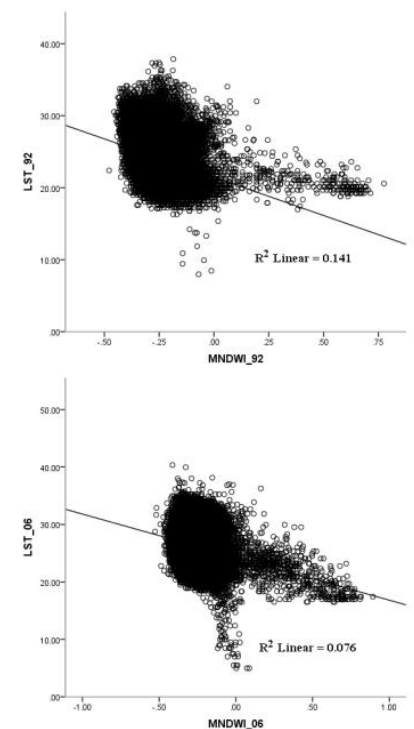

(c)

Fig.6. Relations between (a) NDVI and LST; (b) NDBI and LST; and (c) MNDWI and LST.

Like Zhou \& Wang (2011), scatter plots of LST and remote sensing indices were used to assess the effectiveness of each index in modeling LST in 1992 and 2006 (Fig. 6). The all results indicated statistically significance correlation $(P<0.01)$ between LST and remote sensing indices. Although the study area and the data processing method were slightly different, very similar scatter plots and correlation results were obtained by comparing our study with Zhou and Wang Zhou \& Wang (2011). The differences of results could be caused by clouds that we did not deal with and manner of sampling procedure, which sampled not only built-up land and vegetated pixels, but also many water bodies. Clouds and water body pixels always had low values in these three variable images (LST, NDVI and NDBI), and therefore caused weak correlations between them.

\subsection{Relationship among NDVI, NDBI, NDBaI, NDWI, MNDWI and LST}

Much of the cropland in the study region had been unplanted or ill-managed, and at the same time, forest and grassland had degraded seriously in the process of urbanization. Checked from the high resolution image in Google Earth, we found that high-temperature regions located in the urban, residential areas, villages, towns and farmland areas (not yet planted crops). On the contrary, the lowtemperature regions mainly located in the water and high density vegetation areas. The dense natural vegetation canopy in rural areas reduced the surface radiant temperature which led to relatively low LST values (Zhang, Odeh, \& Han, 2009) while bare and semi-bare lands had high LST where significant sensible heat exchange occurred ( $\mathrm{Pu}$ et al., 2006). Previous studies had suggested that land use and land cover changes, especially urban development, would cause a greater contrast in the surface thermal properties. So, we would like to see whether there were quantifiable relationships between LST and these indices (urban surface characteristics). Due to NDBI and NDWI use the same bands of satellite images, they are mutually opposite number. So the NDBI was analyzed only in this study. 
The relationship between remote sensing indices and LST was investigated through correlation analysis (pixel by pixel). The Pearson's correlation coefficients indicated that all of the remote sensing indices were significantly correlated with the LST (Fig. 7, P<0.01). Comparing with other indices, NDBI were more correlated with LST before 2013. The coefficients of determination of LSTs to NDBI for all times were fairly high (minimum R-square value was larger than 0.4 ). For relatively small affected by rainfall and vegetation growing season, the relationship between LST and NDBI index was relatively stable. These results indicated that at pixel-scale, the relationship between LST and NDBI could be expressed using the linear model, but there might be more complicated relationship pattern, which needed to be further studied in future research. Heterogeneous property of land surface might be the reasons that contributed to wider variations of LSTs, especially in region with low vegetation fraction, such as urban area. For example, pixels with same vegetation abundance (or percent impervious surface) might possess varying LSTs, because the emissivities of these pixels might be different due to different cover types, soil moistures or vegetation conditions, etc. (Ma, Kuang, \& Huang, 2010). It was interesting to note that NDBI had the highest correlation coefficient value before 2013, but NDBaI had the highest correlation coefficient value after 2013. The reason might be the difference of data source, because the data before 2013 came from Landsat5 or Landsat7, and the others came from Landsat8. As new launched satellite, the bands division of Landsat8 is different to Landsat5 and Landsat7.

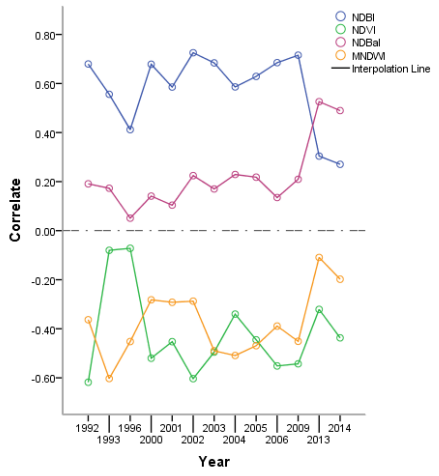

(a)

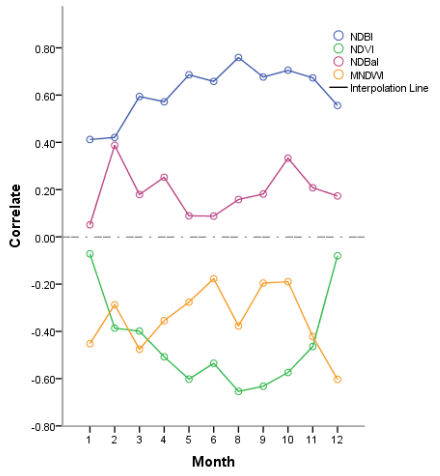

(b)

Fig.7. Correlation coefficients among NDVI, NDBI, NDBaI, NDWI, MNDWI and LST.

\subsection{Regression among NDVI, NDBI, NDBaI, NDWI, MNDWI and LST}

By stepwise regression, a combination of MNDWI and NDVI not only met the requirements of the regression equation well, but also could produce the best regression results (the NDBI and NDVIMNDWI means of R-squared value were 0.37 and 0.47 , respectively). The reason for this might be using the same band in NDBI and other indices calculations, such as NDBI and NDVI all use $\mathrm{R}_{\mathrm{NIR}}$ band, and NDBI and MNDWI all use $\mathrm{R}_{\mathrm{MIR}}$ band. In contrast, NDVI and MNDWI use completely different bands to calculate, so they could get the best combination of regression expression and regression results. NDVI and MNDWI were substantially complementary to each other (Fig. 8). When one was small, another became large. These results implied that at pixel-scale, the relationship between LST and NDVI-MNDWI could be expressed using the linear regression model. From this we could infer that there was a close relationship between LST and vegetation cover and surface moisture. In addition, it was interesting to note that the regression coefficient of determination value (R-square) in 2013 was lower than NDBaI's correlation coefficient. The reason for this was probably persistent drought in 2013 and it ended in 2014, besides data came from Landsat8. All above results suggested that both NDBI and NDVI-MNDWI were acceptable indicators of LST, but NDVI-MNDWI was supposed to a better one. 


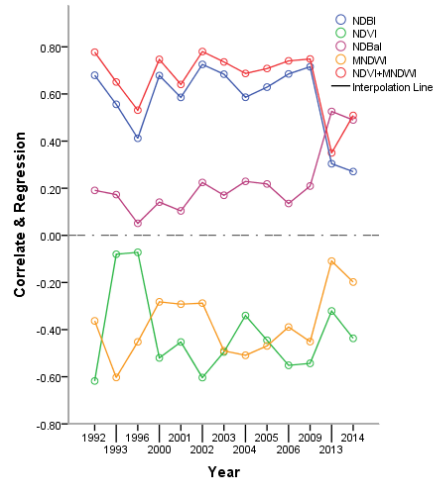

(a)

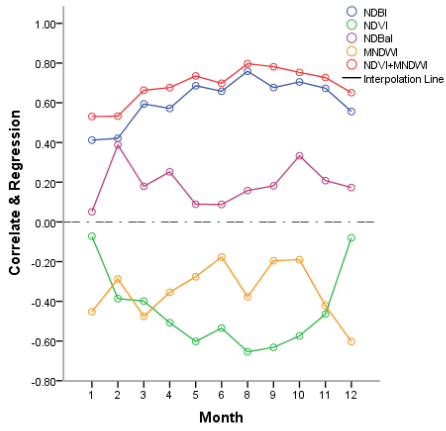

(b)

Fig.8. Regression coefficient between LST and NDVI-MNDWI.

\subsection{UHI intensity}

There was a remarkable positive correlation between UHI intensity of MNDVI and LNDVI, and the correlation coefficient was $0.773(\mathrm{P}<0.01)$. The relationships between UHI intensity and meteorological conditions were investigated through correlation analysis (time by time). The Pearson's correlation coefficients indicated that UHI intensity of MNDVI was significantly correlated with the mean monthly humidity and wind speed $(\mathrm{P}<0.01)$, and the correlation coefficients were -0.601 and 0.613 respectively. Nevertheless humidity was significantly correlated with wind speed $(\mathrm{P}<0.01)$, and the correlation coefficient was -0.726 . It has been known that the UHI intensity can vary with the change of seasons, and that human behaviours would influence the UHI intensity interpretation of land use/cover types (Chen et al., 2006; Zhang, Odeh, \& Han, 2009). Interestingly, the highest UHI intensity appeared in the April of these years from 1992 to 2014 (Fig. 9 (a)), and at the same time, April's LST was also relatively high (Fig. 5 (b)). Nevertheless, UHI intensity changed not only with the seasons, but also over time in this study (Fig. 9). The UHI intensity (represented by LST) had a decreasing trend from 2000 to 2014 (Fig. 9 (b)) using the data in April, althought the regression results were not significant.

The LST is the temperature of land surface, it is closely related to surface moisture and shading degree of ground (Chen, Li, \& Wang, 2015). While the temperature increased greatly in April (Fig. 10 (a), (c)), the sunshine hours in April (Fig. 10 (b)) was also the longest. So the solar radiation increased rapidly from the beginning of April. Otherwise, because the major rainfall months were from April to October in Kunming (Fig. 10 (a)), April was the dry-wet transition month (Han, Chen, \& Feng, 2015). The surface moisture was still relatively low at the beginning of the April (Fig. 10 (b)). Therefore, the land surface temperature was still high. However, at the end of April, relatively large amount of precipitation accompanied with temperature rising, not only greatly increased surface moisture, but also increased the vegetation cover for the growth period. Moreover, the farmland that had violent intensity was planted with the crops after April. Thereby, the intensity of LST reduced greatly in study area.

In addition, from the correlation analysis, we could also know that the greater the wind speed, the lower the surface humidity. While the lower the humidity will lead to greater heat island intensity. In summary, the results suggested that the heat island intensity was mainly affected by solar radiation, surface cover type, wind speed, humidity and other environmental factors. 


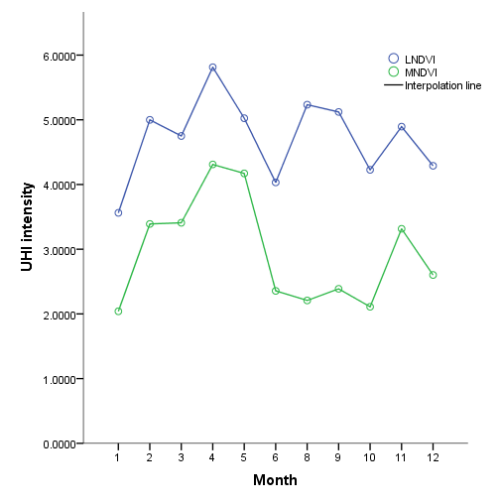

(a)

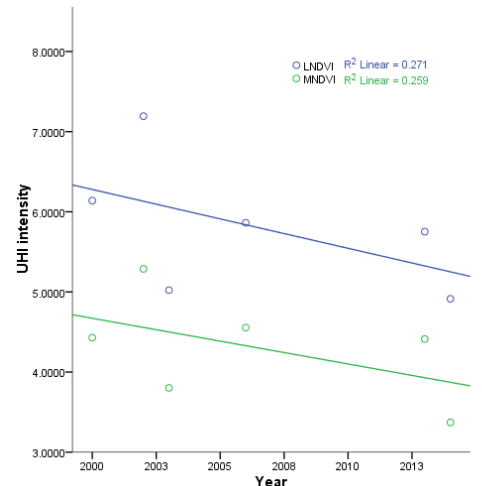

(b)

Fig.9. UHI Intensity of year and month

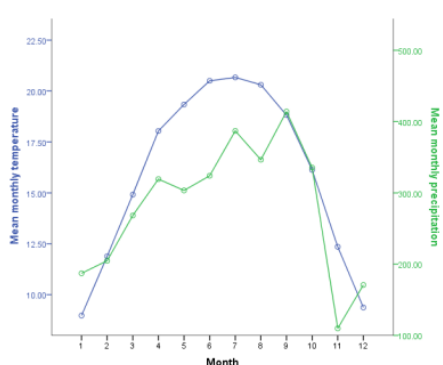

(a)

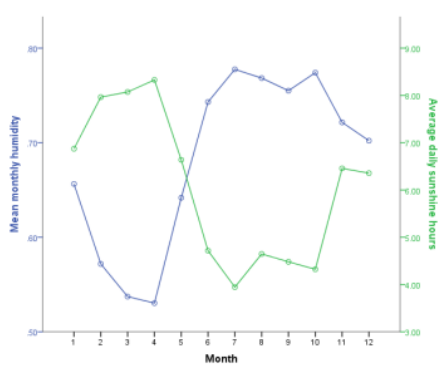

(b)

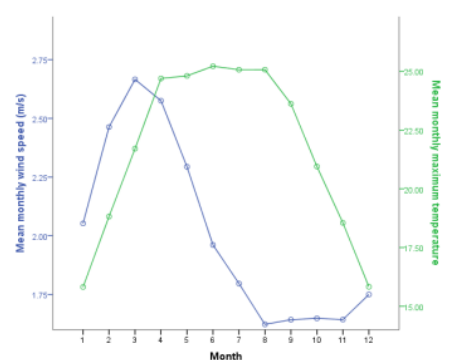

(c)

Fig.10. Meteorological conditions

\subsection{UHI intensity index}

There was a remarkable positive correlation between UHI intensity index of MNDVI and LNDVI, and the correlation coefficient was $0.759(\mathrm{P}<0.01)$. The relationships between UHI intensity index and meteorological conditions were investigated through correlation analysis (time by time). The Pearson's correlation coefficients indicated that UHI intensity indexes of MNDVI and LNDVI were significantly correlated with the mean monthly temperature $(\mathrm{P}<0.01)$, and the correlation coefficients were -0.75 and -0.566 respectively. At the same time, the UHI intensity indexes of MNDVI and LNDVI were significantly correlated with the mean monthly precipitation $(\mathrm{P}<0.05)$, and the correlation coefficient were -0.536 and -0.496 respectively. Similar to the trend of UHI intensity, the UHI intensity index also decreased from 2000 to 2014 (Fig. 11 (b)), but the downward trend was stronger than that of UHI intensity, althought the regression results were not significant too.

The varieties of UHI intensity and UHI intensity index were difference completely (Fig. 9 (a), Fig. 11 (a)). The UHI intensity index figures were upturned relatively large at the beginning and end of time, besides the changing of position and sharpness of the peak. It was worth noting that the lowest intensity index appeared in June. With the beginning of the rainy season in April, the monthly mean temperature gradually increased. The monthly average temperature was higher in June, July and August, and reached the highest in July (Fig. 10 (a)). In particular, the mean monthly maximum temperature kept higher from April to August and reached the highest in June (Fig. 10 (c)). At the same time, with the gradual increase in rainfall, humidity gradually increased, while the sunshine hours gradually reduced (Fig. 10 (b)). The increases of the temperature and the humidity not only reduced the temperature differences between the different land surface types (the molecules of intensity index calculation), but also increased the temperature of HNDVI (the denominator of intensity index calculation). Which eventually led to the UHI intensity index in June became the lowest.

In addition, although the rainfall in June was less than in August, the average monthly temperature and maximum temperature in June was higher than in August (Fig. 10 (a), (c)). From the correlation coefficient and confidence range, we could see that the impact of temperature on the UHI intensity index was greater than the precipitation. So the UHI intensity index in June was lower than in August. Due to data acquisition restrictions, we did not get the data for July. But according to the same reason, we could conclude that because the July's temperature was higher than the June and the July's rainfall 
was much greater than the June, the UHI intensity index in July should not be higher than in June, and might reach the lowest. The spatial distribution of the intensity index greater than 0.5 in the region remained essentially unchanged, and they mainly concentrated in the north, northeast, southeast and southwest (Fig. 12). At the same time, these areas could be considered as key areas where the UHI was focused on elimination. To sum up, the results suggested that the heat island intensity index was mainly affected by temperature, precipitation, solar radiation, land surface cover type and other environmental factors.

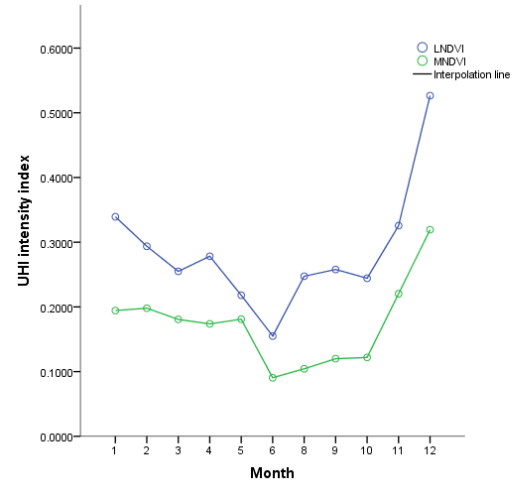

(a)

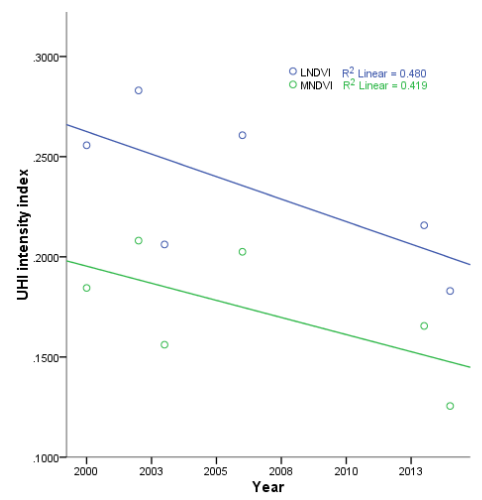

(b)

Fig.11. UHI intensity index of year and month

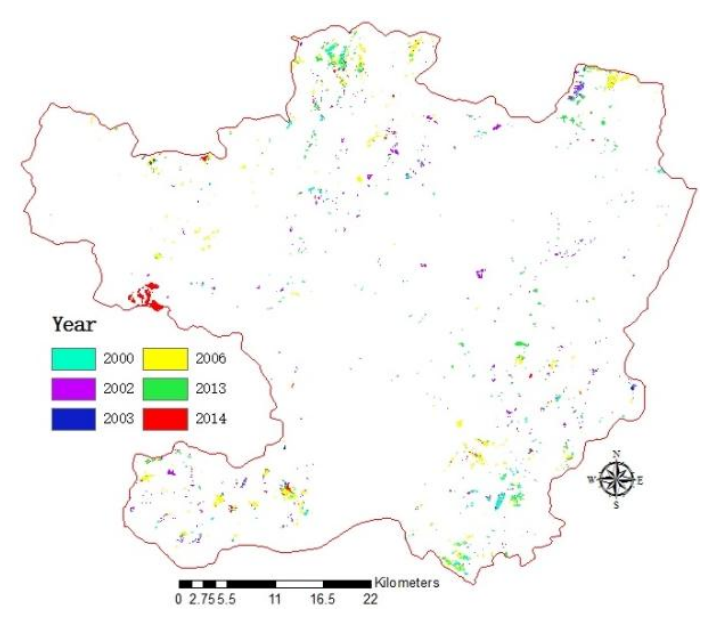

Fig.12. UHI intensity index greater than 0.5 in April

\section{Conclusions}

In this study we investigated the relationships among NDVI, NDBI, NDBaI, NDWI, MNDWI and LST in Kunming city, and several conclusions were made: (1) the reasonably high correlation between LST and NDVI-MNDWI in the most years asserted that the NDBI was a good index of LST, while NDVI-MNDWI was a better one; (2) UHI intensity (represented by LST) got the highest value in April, while the lowest UHI intensity index emerged in June; (3) the regions of intensity index greater than 0.5 were unchanged essentially and these regions could be considered as key areas where the UHI was focused on elimination; (4) the heat island intensity was mainly affected by temperature, solar radiation (sunshine hours), land surface cover type, precipitation, wind speed, humidity and other environmental factors; (5) the heat island intensity index was mainly affected by temperature, precipitation, solar radiation, land surface cover type and other environmental factors.

All the analyses in this study were based on the interpretation of remote sensing images, and results showed that remote sensing image was ideal for analyzing UHI. But the variations of correlations coefficients and multiple coefficients of determination suggested that the change of LST should be influenced by NDBI, NDVI and MNDWI, and other factors. In future, several works need to be 
focused. Firstly, long time series data of Landsat8 will be needed to verify our conclusions. Secondly, high spatial resolution image and surface reflectance image need to be fused to better determine the type of surface objects. Thirdly, more remote sensing indices need to be further studied. Fourthly, the retrieval method of temperature needs to be improved to reduce the influence of thin cloud and inhomogeneous atmosphere condition. Fifthly, the spatial arrangement of land use/cover will be optimized based on ant colony algorithm and GIS/RS. Finally, the errors caused by different conditions need to be more accurately estimated or removed.

\section{Acknowledgements}

This work was supported by the National Natural Science Foundation of China (Nos. 61262070).We thank the USGS and the Remote Sensing Data Sharing Center of China for providing Landsat data. We are indebted to several local environmental agencies and groups who collected and provided meteorological data.

\section{References}

Artis, D.A., \& Carnahan, W.H. (1982). Survey of emissivity variability in thermography of urban areas. Remote Sensing of Environment ,12,313-329.

Cai, H. (2007). Land use change comparative study of Kunming City based on remote sensing. Guizhou Science, 25, 178-184.

Carlson, T.N., \& Ripley, D.A. (1997). On the relation between NDVI, fractional vegetation cover, and leaf area index. Remote Sensing of Environment, 62, 241-252.

Chen, D. \& Brutsaert, W. (1998). Satellite-sensed distribution and spatial patterns of vegetation parameters over a tallgrass prairie. Journal of the Atmospheric Sciences , 55, 1225-1238.

Chen, X., Li, L., \& Wang, J. (2015). Heat Island Effect Mitigation by Urban Green Space System: A Case Study of Taizhou City. Ecology and Environmental Sciences , 24 (4), 643-649.

Chen, X., Zhao, H., Li, P. \& Yin, Z. (2006). Remote sensing image-based analysis of the relationship between urban heat island and land use/cover changes. Remote Sensing of Environment, 104, 133-146.

Choudhury, B.J., Ahmed, N.U., Idso, S.B., Reginato, R.J., \& Daughtry, C.S.T. (1994). Relations between evaporation coefficients and vegetation indices studied by model simulations. Remote Sensing of Environment, 50, 1-17.

Debbage, N., and Shepherd, J. M. (2015). The urban heat island effect and city contiguity. Computers, Environment and Urban Systems, 54, 181-194.

Deng, C., and $\mathrm{Wu}, \mathrm{C}$. (2013). Examining the impacts of urban biophysical compositions on surface urban heat island: A spectral unmixing and thermal mixing approach. Remote Sensing of Environment, 131, 262-274.

Feng, H.H., Zhao, X.F., Chen, F., \& Wu, L.C. (2014). Using land use change trajectories to quantify the effects of urbanization on urban heat island. Advances in Space Research, 53, 463-473.

Gao, B.C. (1996). NDWI-A normalized difference water index for remote sensing of vegetation liquid water from space. Remote Sensing of Environment, 58, 257-266.

Ghosh, A., \& Joshi, P.K. (2014). Hyperspectral imagery for disaggregation of land surface temperature with selected regression algorithms over different land use land cover scenes. ISPRS Journal of Photogrammetry and Remote Sensing, 96, 76-93.

Gutman, G., \& Ignatov, A. (1998). The derivation of the green vegetation fraction from NOAA/AVHRR data for use in numerical weather prediction models. International Journal of Remote Sensing, 19, 1533-1543.

Han, X., Chen, X., \& Feng, Lian. (2015). Four decades of winter wetland changes in Poyang Lake based on Landsat observations between 1973 and 2013. Remote Sensing of Environment, 156, 426-437.

Knorn, J., Rabe, A., Radeloff, V.C., Kuemmerle, T., Kozak, J., \& Hostert, P. (2009). Land cover mapping of large areas using chain classification of neighboring Landsat satellite images. Remote Sensing of Environment, 113, 957-964.

Li, J., Song, C., Cao, L., Zhu, F., Meng, X., \& Wu, J. (2011). Impacts of landscape structure on surface urban heat islands: a case study of Shanghai, China. Remote Sensing of Environment, 115, 32493263. 
Li, J.J., Wang, X.R., Wang, X.J., Ma, W.C., \& Zhang, H. (2009). Remote sensing evaluation of urban heat island and its spatial pattern of the Shanghai metropolitan area, China. Ecological Complexity, 6, 413-420.

Li, X., Zhou, W., \& Ouyang, Z. (2013). Relationship between land surface temperature and spatial pattern of greenspace: What are the effects of spatial resolution? Landscape and Urban Planning ,114, 1-8.

Lucas, I.F.J., Frans, J.M., \& Wel, V.D. (1994). Accuracy assessment of satellite derived land-cover data: a review. Photogrammetric Engineering and Remote Sensing, 60, 410-432.

Luo, J. \& Wei, Y.H.D. (2009). Modeling spatial variations of urban growth patterns in Chinese cities: The case of Nanjing. Landscape and Urban Planning, 91, 51-64.

Ma, Y., Kuang, Y.Q., \& Huang, N.S. (2010). Coupling urbanization analyses for studying urban thermal environment and its interplay with biophysical parameters based on TM/ETM+ imagery. International Journal of Applied Earth Observation and Geoinformation, 12(2), 110-118.

Martilli, A. (2014). An idealized study of city structure, urban climate, energy consumption, and air quality. Urban Climate, 10, 430-446.

Oke, T.R. (1982). The energetic basis of the urban heat island. Quarterly Journal of the Royal Meteorological Society, 108, 1-24.

Rouse, J.W., Haas, R.H., Schell, J.A., \& Deering, D.W. (1973). Monitoring vegetation systems in the Great Plains with ERTS. In: Proceedings of the Third ERTS Symposium, Washington, DC, pp. 309-317.

Roy, A.H., Dybas, A.L., Fritz, K.M. \& Lubbers, H.R. (2009). Urbanization affects the extent and hydrologic permanence of headwater streams in a midwestern US metropolitan area. Journal of the North American Benthological Society, 28, 911-928.

Pu, R., Gong, P., Michishita, R. \& Sasagawa, T. (2006). Assessment of multi-resolution and multisensor data for urban surface temperature retrieval. Remote Sensing of Environment, 104, 211225.

Snyder, W.C., Wan, Z., Zhang, Y., \& Feng, Y.Z. (1998). Classification based emissivity for land surface temperature measurement from space. International Journal of Remote Sensing, 19, 27532774.

Sobrino, J.A., Jime'nez-Muñoz, J.C., \& Paolini, L. (2004). Land surface temperature retrieval from LANDSAT TM 5. Remote Sensing of Environment, 90, 434-440.

Sobrino, J.A., Raissouni, N., \& Li, Z.L. (2001). A comparative study of land surface emissivity retrieval from NOAA data. Remote Sensing of Environment, 75, 256-266.

Van, T. \& Bao, H. (2010). Study of the impact of urban development on surface temperature using remote sensing in Ho Chi Minh City, northern Vietnam. Geographical Research, 48, 86-96.

Vlassova, L., Perezcabello, F., Nieto, H., Martin, P., Riano, D. \& La Riva, J. (2014). Assessment of Methods for Land Surface Temperature Retrieval from Landsat-5 TM Images Applicable to Multiscale Tree-Grass Ecosystem Modeling. Remote Sensing, 6, 4345-4368.

Weng, Q., Lu, D. \& Schubring, J. (2004). Estimation of land surface temperature-vegetation abundance relationship for urban heat island studies. Remote Sensing of Environment, 89, 467483.

Wu, H., Ye, L.P., Shi, W.Z., \& Clarke, K.C. (2014). Assessing the effects of land use spatial structure on urban heatislands using HJ-1B remote sensing imagery in Wuhan, China. International Journal of Applied Earth Observation and Geoinformation, 32, 67-78.

Xiao, R., Ouyang, Z., Zheng, H., Li, W., Schienke, E.W., \& Wang, X. (2007). Spatial pattern of impervious surfaces and their impacts on land surface temperature in Beijing, China. Journal of Environmental Sciences, 19(2), 250-256.

$\mathrm{Xu}, \mathrm{H}$. (2006). Modification of normalized difference water index (NDWI) to enhance open water features in remotely sensed imagery. International Journal of Remote Sensing, 27, 3025-3033.

$\mathrm{Xu}, \mathrm{H}$. (2008a). A new index for delineating built-up land features in satellite imagery. International Journal of Remote Sensing, 29 (14), 4269-4276.

$\mathrm{Xu}, \mathrm{H}$. (2008b). A new remote sensing index for fastly extracting impervious surface information. Geomatics and Information Science of Wuhan University, 33, 1150-1153.

Xu, L.Y., Xie, X.D., \& Li, S. (2013). Correlation analysis of the urban heat island effect and the spatial and temporal distribution of atmospheric particulates using TM images in Beijing. Environmental Pollution, 178, 102-114.

Yang, L., Cao, Y.G., Zhu, X.H., Zeng, S.H., Yang, G.J., He, J.Y., \& Yang, X.C. (2014). Land surface temperature retrieval for arid regions based on Landsat-8 TIRS data: a case study in Shihezi, Northwest China. Journal of Arid Land, 6(6), 704-716. 
Yang, L., Xian, G., Klaver, J.M., \& Deal, B. (2003). Urban lad-cover change detection through subpixel imperviousness mapping using remotely sensed data. Photogrammetric Engineering and Remote Sensing, 69 (9), 1003-1010.

Yu, X., Guo, X., \& Wu, Z. (2014). Land Surface Temperature Retrieval from Landsat 8 TIRSComparison between Radiative Transfer Equation-Based Method, Split Window Algorithm and Single Channel Method. Remote Sensing, 6, 9829-9852.

Yuan, F., \& Bauer, M.E. (2007). Comparison of impervious surface area and normalized difference vegetation index as indicators of surface urban heat island effects in Landsat imagery. Remote Sensing of Environment, 106, 375-386.

Zha, Y., Gao, J., \& Ni, S. (2003). Use of normalized difference built-up index in automatically mapping urban areas from TM imagery. International Journal of Remote Sensing, 24 (3), 583659.

Zhang, Y.S., Odeh, I.O.A., \& Han, C.F. (2009). Bi-temporal characterization of land surface temperature in relation to impervious surface area NDVI and NDBI, using a sub-pixel image analysis. International Journal of Applied Earth Observation and Geoinformation, 11 (4), 256264.

Zheng, G., Lu, M., Zhang, T., Liu, G., \& Ke, C. (2010). The impact of difference of land surface emissivity on the land surface temperature retrieval in Jinan City. Journal of Shandong Jianzhu University, 25(5), 519-523.

Zhou, X., \& Wang, Y. (2011). Dynamics of Land Surface Temperature in Response to Land Use/Cover Change. Geographical Research, 49(1), 23-36. 